\title{
Characterization of the Affective Norms for English Words by discrete emotional categories
}

\author{
Ryan A. Stevenson \\ Indiana University, Bloomington, Indiana \\ JoSEPH A. MIKELS \\ Cornell University, Ithaca, New York \\ AND \\ Thomas W. JaMes \\ Indiana University, Bloomington, Indiana
}

\begin{abstract}
The Affective Norms for English Words (ANEW) are a commonly used set of 1,034 words characterized on the affective dimensions of valence, arousal, and dominance. Traditionally, studies of affect have used stimuli characterized along either affective dimensions or discrete emotional categories, but much current research draws on both of these perspectives. As such, stimuli that have been thoroughly characterized according to both of these approaches are exceptionally useful. In an effort to provide researchers with such a characterization of stimuli, we have collected descriptive data on the ANEW to identify which discrete emotions are elicited by each word in the set. Our data, coupled with previous characterizations of the dimensional aspects of these words, will allow researchers to control for or manipulate stimulus properties in accordance with both dimensional and discrete emotional views, and provide an avenue for further integration of these two perspectives. Our data have been archived at www.psychonomic.org/archive/.
\end{abstract}

Affect has been studied using a wide range of methodologies, procedures, and tasks. In addition, a wide range of stimuli have been used in the study of emotion, including pictures of facial expressions, static pictures of emotional scenes, video clips, imagery inductions, nonlinguistic verbalizations, environmental sounds, prosody, and spoken and written words. One of the largest word sets is the Affective Norms for English Words (ANEW; Bradley \& Lang, 1999), a set of 1,034 English words including verbs, nouns, and adjectives. ANEW has been characterized along the dimensions of valence, arousal, and dominance, as well as by word frequency. Dimensional norms, however, are only one of two general types of norms that are useful for characterizing affective stimuli. The other major approach involves classifying the stimulus set along emotional category lines. Providing these complementary data for the ANEW stimulus set would potentially open many new possibilities to researchers.

Dimensional theories of affect have been shown to be most powerful when models include two categories, valence and arousal (Mehrabian \& Russel, 1974; Smith \& Ellsworth, 1985; Yik, Russell, \& Barrett, 1999). Empirical applications of the dimensional model using words as stimuli have been successfully used in many studies. Behaviorally, positive and negative words elicit lower reac- tion times and higher accuracy than neutral words (Ali \& Cimino, 1997; Borod, Andelman, Obler, Tweedy, \& Welkowitz, 1992; Eviatar \& Zaidel, 1991; Graves, Landis, \& Goodglass, 1980; Inaba, Nomura, \& Ohira, 2005; Kuchinke et al., 2005), and emotional words have been shown to induce emotional priming (Brouillet \& Syssau, 2005; Carroll \& Young, 2005; Van Strien \& Morpurgo, 1992). Distinct neural correlates have been found that respond to changes in the valence level of words (Cato et al., 2004; Fossati et al., 2003; Kuchinke et al., 2005; Lewis, Critchley, Rotshtein, \& Dolan, 2007), and these have been differentiated from neural correlates for which activation varies according to arousal (Lewis et al., 2007). Likewise, patients with brain lesions have shown specific deficits in responses to words and sentences of specific valences (Anderson \& Phelps, 2001; Borod et al., 1992).

Models of emotion based on discrete categories have also proved useful in empirical studies. These studies have focused on the use of facial expressions (Ekman, 1993) and static pictures (Bradley, Codispoti, Cuthbert, \& Lang, 2001; Lang, Greenwald, Bradley, \& Hamm, 1993; Mikels et al., 2005) to show differences in facial electromyography, heart rate, and electrodermal measures between different discrete emotions. Studies of brain-damaged patients have also revealed impairment in emotional recog-

R.A. Stevenson, stevenra@indiana.edu 
nition of particular discrete emotions (Scott et al., 1997), suggesting that discrete emotions have at least partially distinct neural correlates. The discrete categories of happiness, fear, disgust, sadness, and anger have been shown to be universal across cultures and ages (Levenson, 2003), adding support to the categorical model of emotion.

Both the dimensional and categorical approaches have been shown to be effective characterizations of affect, but they are not mutually exclusive. Current views of the dimensional model hold that positive and negative valence systems can be viewed as appetitive and defensive systems, respectively (Bradley, Codispoti, Cuthbert, \& Lang, 2001; Bradley, Codispoti, Sabatinelli, \& Lang, 2001), and arousal is the intensity of the activation. Further, stimuli such as the International Affective Picture System (IAPS; Lang, Bradley, \& Cuthbert, 2005), which were originally described in accord with the dimensional approach, have been shown to produce different responses in skin conductance, startle reflex, and heart rate depending on their emotional category (Bradley, Codispoti, Cuthbert, \& Lang, 2001; Bradley, Codispoti, Sabatinelli, \& Lang, 2001). Individual stimuli from the IAPS have also been rated on emotional categories, with individual pictures showing category-specific responses (Mikels et al., 2005). Thus, whereas a dimensional approach can describe a number of broad features of emotion, and the categorical approach can capture more discrete emotional responses, the two can also be used in combination to supply experimenters with a more complete view of affect.

To provide a discrete categorical characterization of ANEW, we collected categorical ratings of the entire set on happiness, sadness, fear, disgust, and anger, on the basis of the set of cross-culturally universal basic emotions (Ekman, 1993; Levenson, 2003). Given the usefulness of discrete emotional categories with other stimulus types, it follows that studies using words as stimuli would likewise benefit from a categorical characterization, in addition to the already-established dimensional norms. With such data, the ANEW stimulus set will be an excellent tool for further integrating these two views of emotion and will provide a means to investigate both views with one set of stimuli.

\section{METHOD}

\section{Participants}

Participants included 299 native English speakers (125 male, 174 female, mean age 21 years), who received course credit for participation. The experimental protocol was approved by the Indiana University Committee for the Use of Human Subjects in Research.

\section{Design and Procedure}

The ANEW database (1,034 words) was separated into eight groups of 129-130 words. Participants rated two of the eight groups of ANEW words on five discrete emotions on a scale of $1-5$, with 1 being not at all and 5 being extremely. Five independent ratings were made for each word according to this $1-5$ scale, one rating for each of the five emotional categories. Emotional categories were presented in the following order: happiness, anger, sadness, fear, and disgust. Word order was counterbalanced across groups.

\section{RESULTS}

Means and standard deviations of the five ratings were calculated for each word individually (see the archived supplemental materials). Prior to analysis, words were separated into groups of negative (rating $<5$ ) and positive (rating $>5$ ) valence. Regressions were run using the discrete emotional category ratings collected for use in predicting the previous ratings for valence, arousal, and dominance (Bradley \& Lang, 1999). Regressions using the dimensional ratings to predict emotional category ratings were also run.

Six regressions were run using the five emotional category ratings to predict valence, arousal, and dominance within both the positive and negative groups of words. Standardized $\beta$ coefficients were calculated for all five emotional categories (Table 1). With negative words,

Table 1

\begin{tabular}{|c|c|c|c|c|c|c|}
\hline & \multicolumn{2}{|c|}{$\begin{array}{l}\text { Predicting } \\
\text { Valence }\end{array}$} & \multicolumn{2}{|c|}{$\begin{array}{c}\text { Predicting } \\
\text { Arousal }\end{array}$} & \multicolumn{2}{|c|}{$\begin{array}{l}\text { Predicting } \\
\text { Dominance }\end{array}$} \\
\hline & $\beta$ & $t$ & $\beta$ & $t$ & $\beta$ & $t$ \\
\hline \multicolumn{7}{|c|}{ Negative Valence } \\
\hline Happiness & .291 & $8.91^{* *}$ & .050 & 1.36 & .136 & $2.93^{*}$ \\
\hline Anger & -.044 & 1.27 & .492 & $12.59^{* *}$ & .369 & $7.49^{* *}$ \\
\hline Sadness & -.515 & $13.80^{* *}$ & -.309 & $7.33^{* *}$ & -.625 & $11.75^{* *}$ \\
\hline Fear & .020 & 0.58 & .670 & $17.11^{* *}$ & -.144 & $2.91^{*}$ \\
\hline Disgust & -.243 & $8.27^{* *}$ & -.042 & 1.27 & .041 & 0.98 \\
\hline \multicolumn{7}{|c|}{ Positive Valence } \\
\hline Happiness & .890 & $45.40^{* *}$ & .649 & $19.75^{* *}$ & .601 & $16.60^{* *}$ \\
\hline Anger & -.020 & 0.73 & .139 & $2.984^{*}$ & .153 & $2.98^{*}$ \\
\hline Sadness & -.110 & $4.24^{* *}$ & -.287 & $6.57^{* *}$ & -.305 & $6.34^{* *}$ \\
\hline Fear & .116 & $4.95^{* *}$ & .441 & $11.26^{* *}$ & .125 & $2.88^{*}$ \\
\hline Disgust & -.035 & 1.55 & .051 & 1.36 & .042 & 1.00 \\
\hline
\end{tabular}

Note $-\beta$ values, $t$ scores, and significance levels are shown for each emotional category with respect to each emotional dimension. ${ }^{*} p<.005 .{ }^{* *} p<.001$. 
valence was strongly related to sadness, happiness, and disgust; arousal was strongly related to fear, anger, and sadness; dominance was strongly related to sadness and anger, and significantly related to fear and happiness. With positive words, valence was strongly related to happiness, fear, and sadness; arousal was strongly related to happiness, fear, and sadness, and significantly related to anger; dominance was strongly related to happiness and sadness, and significantly related to anger and fear.

The regressions using the dimensional ratings to predict emotional category ratings were similar to the previous regressions, with a lack of homogeneity in the ability of categorical ratings to predict dimensional ratings. (Beta weights and statistical analysis are available in the archived supplemental materials.)

Responses were analyzed according to sex for each emotional category and word. Means and standard deviations were calculated for female and male ratings independently (see the archived supplemental materials). Females gave average ratings that were significantly greater than males for happiness (mean difference $=0.14 ; t=12.31, p<.0001$ ), anger (mean difference $=0.07 ; t=10.53, p<.0001$ ), sadness (mean difference $=0.10 ; t=12.00, p<.0001$ ), fear (mean difference $=0.24 ; t=22.60, p<.0001$ ), and disgust (mean difference $=0.20 ; t=21.12, p<.0001$ ).

To further investigate these apparent sex differences, effect sizes and paired-samples correlations for sex differences were calculated for happiness $(d=.12, r=.97)$, anger $(d=.11, r=.95)$, sadness $(d=.25, r=.95)$, fear $(d=.29, r=.94)$, and disgust $(d=.14, r=.94)$.

We ran $t$ tests on ratings for each individual word for all five discrete emotional categories. A limited number of words showed sex differences for happiness $(11.04 \%)$, anger (4.07\%), sadness (13.07\%), fear (13.07\%), and disgust $(5.52 \%)$, for a total of $9.35 \%$ of ratings showing sex differences.

\section{DISCUSSION}

The data from this study provide categorical data that will allow ANEW to be used in studies of emotional categories, and also will provide a means of investigating the association of the dimensional and categorical approaches to the study of affect. The heterogeneity of effects that each emotional category has on different dimensional attributes of the stimuli highlights the importance of using categorical data both independently and as a supplement with dimensional data.

\section{Emotional Categories and Dimensions}

In an effort to ascertain whether the information collected by emotional categories represented the same emotional information described by the previous dimensional ratings, a regression was run using the categorical data of each word to predict the dimensional data, and vice versa. Each emotional dimension was driven by unique pairings of emotional categories. For example, within the set of negative words, valence was driven primarily by sadness, and to a lesser extent by disgust and happiness, whereas arousal was driven primarily by fear and anger, and to a lesser extent by sadness. Surprisingly, many emotional categories did not have any significant impact on one or two of the previously measured dimensions. Anger and fear did not significantly predict valence, whereas happiness and disgust did not predict arousal within negative words. In addition, there were differences in which categories had a strong effect, depending on whether the words were positive or negative. This can be seen in the manner that happiness predicted arousal; for negative words, happiness had no significant effect, but for positive words, happiness was the strongest predictor of arousal. This lack of homogeneity in the effects of categories on affective dimensions reinforces the need to reconsider the dimensional and categorical approaches as independent perspectives, as well as putting stress on the need for stimuli that have been characterized according to both.

The effects of specific discrete emotional categories and emotional dimensions, and the relationships between these two descriptive methods, are still unclear. One possibility is that each discrete emotional category affects valence, arousal, and dominance in a unique and measurable way. Another possibility is that the scales on which dimensions are measured cause the measurements to be more dependent on some emotional categories than on others. For example, it is possible that the pleasure SelfAssessment Manikin that Bradley and Lang (1999) used to determine the valance of words is more sensitive to happiness and sadness than it is to anger. Future work is needed to explore these possibilities.

Regressions using the dimensional ratings to predict emotional category ratings were also run. Results were similar to those of the previous regression, with a lack of homogeneity in the ability of dimensional ratings to predict categorical ratings. Again, the motive behind this analysis has not been to support a theoretical framework, but to emphasize the point that these two sets of ratings impart different information about the stimuli. Emotional categories cannot be extrapolated from the dimensional information, and conversely, dimensional information cannot be extrapolated from the emotional categories. As such, it is important to take both the dimensional and categorical sets of descriptive data into consideration when choosing stimuli, if one wishes to control for as many variables as possible.

\section{Sex Differences}

Sex differences found in the ratings of discrete emotional categories were minimal. Although the differences for mean ratings of males and females were significant for each category, the actual difference was minute, with an overall mean difference of only 0.15 , which is well within even the lowest standard deviation for any emotional category (0.68). The small effect sizes and extremely large paired-samples correlations show that these findings of significant differences in each category do not reflect a large sex difference, but are mainly due to the large number of participants.

Individual words showed a low rate of sex differences, with an average of $9.35 \%$ across emotional categories. This level is similar to the percentage of positive and neg- 
ative IAPS pictures with sex differences $(9.21 \%)$ found by Mikels and colleagues (2005), and it is also consistent with Bradley, Codispoti, Sabatinelli, and Lang's (2001) findings of minimal sex differences with IAPS pictures. That being said, there was a small group of words that differed across sex in many of the discrete emotional categories. A single word ( $\mathrm{man}$ ) differed across all five emotional categories, and 5 words (abuse, bomb, destroy, pistol, and shotgun) differed in four categories, and a further 23 words differed in three categories. All words with sex differences have been noted in the archived supplemental materials, with category-specific information included.

Although our results support previous findings of minimal differences across the sexes, there are a number of outlying words that do produce differences. Our data are of a descriptive nature, and as such should not be used to infer what causes these slight differences. Our characterization of ANEW allows researchers an avenue to pursue future studies of these differences and any theoretical implications they may have.

\section{Future Uses}

This categorical characterization of ANEW will provide a means to further study the effects of discrete emotion on behavior and cognition. A number of studies have contrasted the effects of discrete emotions on behavior (Keltner, Ellsworth, \& Edwards, 1993; Lerner \& Keltner, 2001), as well as how different discrete emotions are processed neurologically (Hans, Eckart, \& Hermann, 1997; Morris et al., 1998; Morris et al., 1996; Phillips et al., 1998; Scott et al., 1997); however, stimulus sets that are thoroughly characterized according to discrete emotions are not common, and those normalized along both discrete emotions and emotional dimensions are rare.

Our characterization of ANEW on the basis of discrete categories provides researchers with a means to choose stimuli that are more highly controlled for emotional content. Ratings for each word in ANEW have been archived, including means and standard deviations of each emotion category, and in addition, ratings for individual words differentiated by sex have also been made available, as well as sex differences. Our data will allow researchers to choose words associated with a single emotion or with blended emotions, or to choose undifferentiated words (for an example, see Mikels et al., 2005), as well as providing the flexibility for researchers to choose the confidence level that is appropriate for a given study.

Our data help to provide a more complete characterization of ANEW, which will allow researchers to be more selective and precise in stimulus selection, as well as providing another level on which future data can be analyzed. The previous normalizations of IAPS and the present normalization of ANEW provide an avenue for researchers to further the integration of the categorical and dimensional models of emotion.

\section{AUTHOR NOTE}

This research was supported in part by the Indiana METACyt Initiative of Indiana University. Thanks to Laurel Stevenson and Karin James, as well as Marisa Geoghegan and Jennifer Willingham for their support and insights on this work and the manuscript. Correspondence relating to this article may be sent to R. A. Stevenson, Department of Psychological and Brain Sciences, Indiana University, 1101 East Tenth Street, Room 293, Bloomington, IN 47405 (e-mail: stevenra@indiana.edu).

\section{REFERENCES}

Ali, N., \& Cimino, C. R. (1997). Hemispheric localization of perception and memory for emotional verbal stimuli in normal individuals. Neuropsychology, 11, 114-125.

Anderson, A. K., \& Phelps, E. A. (2001). Lesions of the human amygdala impair enhanced perception of emotionally salient events. Nature, 411, 305-309.

Borod, J. C., Andelman, F., Obler, L. K., Tweedy, J. R., \& WelkoWITZ, J. (1992). Right hemisphere specialization for the identification of emotional words and sentences: Evidence from stroke patients. Neuropsychologia, 30, 827-844.

Bradley, M. M., Codispoti, M., Cuthbert, B. N., \& Lang, P. J. (2001). Emotion and motivation I: Defensive and appetitive reactions in picture processing. Emotion, 1, 276-298.

Bradley, M. M., Codispoti, M., Sabatinelli, D., \& Lang, P. J. (2001). Emotion and motivation II: Sex differences in picture processing. Emotion, 1, 300-319.

Bradley, M. M., \& Lang, P. J. (1999). Affective Norms for English Words (ANEW): Stimuli, instruction manual, and affective ratings (Tech. Report C-1). Gainesville: University of Florida, Center for Research in Psychophysiology.

Brouillet, T., \& Syssau, A. (2005). Connection between the evaluation of positive or negative valence and verbal responses to a lexical decision making task. Canadian Journal of Experimental Psychology, 59, 255-261.

Carroll, N. C., \& Young, A. W. (2005). Priming of emotion recognition. Quarterly Journal of Experimental Psychology, 58A, 1173-1197.

Cato, M. A., Crosson, B., Gökçay, D., Soltysik, D., Wierenga, C., Gopinath, K., ET AL. (2004). Processing words with emotional connotation: An fMRI study of time course and laterality in rostral frontal and retinosplenial cortices. Journal of Cognitive Neuroscience, 16, 167-177.

Ekman, P. (1993). Facial expression and emotion. American Psychologist, 48, 384-392.

Eviatar, Z., \& Zaidel, E. (1991). The effects of word length and emotionality on hemispheric contributions to lexical decision. Neuropsychologia, 29, 415-428.

Fossati, P., Hevenor, S. J., Graham, S. J., Grady, C., Keightley, M. L., Craik, F., \& Mayberg, H. (2003). In search of the emotional self: An fMRI study using positive and negative emotional words. American Journal of Psychiatry, 160, 1938-1945.

Graves, R., Landis, T., \& Goodglass, H. (1980). Laterality and sex differences for visual recognition of emotional and non-emotional words. Neuropsychologia, 19, 95-102.

Hans, P., Eckart, A., \& Hermann, A. (1997). The cortical processing of perceived emotion: A DC-potential study on affective speech prosody. NeuroReport, 8, 623-627.

Inaba, M., Nomura, M., \& OHIRA, H. (2005). Neural evidence of effects of emotional valence on word recognition. International Journal of Psychophysiology, 57, 165-173.

Keltner, D., Ellsworth, P. C., \& Edwards, K. (1993). Beyond simple pessimism: Effects of sadness and anger on social perception. Journal of Personality \& Social Psychology, 64, 740-752.

Kuchinke, L., Jacobs, A. M., Grubich, C., Vo, M. L., Conrad, M., \& HerrmanN, M. (2005). Incidental effects of emotional valence in single word processing: An fMRI study. NeuroImage, 28, 1022-1032.

Lang, P. J., Bradley, M. M., \& Cuthbert, B. N. (2005). International Affective Picture System (IAPS): Affective ratings of pictures and instruction manual (Tech. Report A-6). Gainesville: University of Florida, Center for Research in Psychophysiology.

Lang, P. J., Greenwald, M. K., Bradley, M. M., \& Hamm, A. O. (1993). Looking at pictures: Affective, facial, visceral, and behavioral reactions. Psychophysiology, 30, 261-273.

LERNER, J. S., \& KelTnER, D. (2001). Beyond valence: Toward a model of emotion-specific influences on judgment and choice. Cognition \& Emotion, 14, 473-493.

LeVenson, R. W. (2003). Autonomic specificity and emotion. In R. J. 
Davidson, K. R. Scherer, \& H. H. Goldsmith (Eds.), Handbook of affective sciences (pp. 212-224). Oxford: Oxford University Press.

Lewis, P. A., Critchley, H. D., Rotshtein, P., \& Dolan, R. J. (2007). Neural correlates of processing valence and arousal in affective words. Cerebral Cortex, 17, 742-748.

Mehrabian, A., \& Russell, J. A. (1974). An approach to environmental psychology. Cambridge, MA: MIT Press.

Mikels, J. A., Fredrickson, B. L., Larkin, G. R., Lindberg, C. M., Maglio, S. J., \& Reuter-Lorenz, P. A. (2005). Emotional category data on images from the International Affective Picture System. Behavior Research Methods, 37, 626-630.

Morris, J. S., Friston, K. J., Büchel, C., Frith, C. D., Young, A. W., CALDER, A. J., ET AL. (1998). A neuromodulatory role for the human amygdala in processng emotional facial expressions. Brain, 121, 47-57.

Morris, J. S., Frith, C. D., Perrett, D. I., Rowland, D., Young, A. W., Calder, A. J., \& Dolan, R. J. (1996). A differential neural response in the human amygdala to fearful and happy facial expressions. Nature, $\mathbf{3 8 3}, 812-815$.

Phillips, M. L., Young, A. W., Scott, S. K., Calder, A. J., AnDREW, C., Giampietro, V., ET AL. (1998). Neural responses to facial and vocal expressions of fear and disgust. Proceedings of the Royal Society B, 265, 1809-1817.

Scott, S. K., Young, A. W., Calder, A. J., Hellawell, D. J., AggleTON, J. P., \& JoHNSON, M. (1997). Impaired auditory recognition of fear and anger following bilateral amygdala lesions. Nature, 385, 254-257.

Smith, C. A., \& Ellsworth, P. C. (1985). Patterns of cognitive ap- praisal in emotion. Journal of Personality \& Social Psychology, 48, 813-838.

Van Strien, J. W., \& Morpurgo, M. (1992). Opposite hemispheric activations as a result of emotionally threatening and non-threatening words. Neuropsychologia, 30, 845-848.

Yik, M. S. M., Russell, J. A., \& Barrett, L. F. (1999). Structure of self-reported current affect: Integration and beyond. Journal of Personality \& Social Psychology, 77, 600-619.

\section{ARCHIVED MATERIALS}

The following materials associated with this article may be accessed through the Psychonomic Society's Norms, Stimuli, and Data archive, www.psychonomic.org/archive/.

To access these files, search the archive for this article using the journal name (Behavior Research Methods), the first author's name (Stevenson), and the publication year (2007).

FILE: NAME-200X.zip

DESCRIPTION: The compressed archive file contains two files:

File1.ext, containing

File2.ext, containing

AUTHOR’s E-MAIL ADDRESS: stevenra@indiana.edu.

(Manuscript received November 14, 2006; revision accepted for publication December 27, 2006.) 